\title{
Mediating Asymmetries in Family Communication: Supporting the eInclusion of Older Adults
}

\author{
Francisco J. Gutierrez ${ }^{1}$, Sergio F. Ochoa ${ }^{1}$, and Julita Vassileva ${ }^{2}$ \\ ${ }^{1}$ Computer Science Department, University of Chile \\ Beauchef 851, 3rd Floor \\ Santiago, Chile \\ frgutier, sochoa\}@dcc.uchile.cl \\ ${ }^{2}$ Computer Science Department, University of Saskatchewan \\ 176 Thorvaldson Bldg., 110 Science Place \\ Saskatoon SK S7N 5C9, Canada \\ jivecs.usask.ca
}

\begin{abstract}
.
Background: The rise of mobile Web-based technologies has diversified the mechanisms used by people to socialize, which results in issues in family communication. Among these concerns, the reluctance of older adults to use digital media may cause them social isolation, leading to negative effects in their physical and mental health. Objective: This paper aims to formalize a model to mediate asymmetries in crossgenerational communication and support the eInclusion of older adults.

Methods: We conducted semi-structured interviews to the members of 20 crossgenerational families. Following the grounded theory approach, we identified emerging themes regarding asymmetries in family communication practices when older adults are involved. We then derived and formalized computer-based mediation strategies using a model-driven engineering approach.

Results: We identified three main sources of asymmetries: (1) implicit family agreements in terms of social interaction, (2) capability and preferences for using particular media, and (3) unbalanced socio-affective coupling between the involved parties. The proposed model addresses these asymmetries and provides strategies to coordinate the communication effort of family members with their elders.

Conclusions: By using the proposed model, designers of software that supports family communities can conceive effective mechanisms to coordinate and mediate social communication among cross-generational family members through digital means. This allows the elderly to show a better reaction to digital media, thus facilitating their acceptance and appropriation of information technologies.
\end{abstract}

Keywords: family communication, older adults, asymmetry, model, mediation, social and digital inclusion. 


\section{$1 \quad$ Introduction}

As a society, we experience our lives as much more dynamic than ever, being mainly focused on reaching individual goals [11]. The downside is that time for socializing is reduced [22]. Therefore, people find in social media (e.g. email, social networking services and videoconference) an efficient way to interact with others, because these mechanisms provide ubiquity, flexibility and efficiency.

In the case of intergenerational families, this social interaction paradigm typically produces a communication gap between older adults and the rest of the families [20]. Some of the causes that explain this gap can be found in elders being reluctant to use technology, even for socializing [8]. Consequently, older adults become more and more socially isolated [14]. Although most elders are eager to address this technological shift, they usually fail due to their physical and cognitive limitations produced by the aging process [5, 12]. Therefore, they need support and guidance to face this complex scenario in a pleasant way [26]. Otherwise, technological adoption by older adults dramatically diminishes.

This paper proposes a model of computer-mediated communication strategies to facilitate the eInclusion of elderly people. In order to improve user acceptance, such mediation strategies need to consider the interaction preferences of each party.

Although promoting social interaction among family members is a commendable objective, such interaction must not overwhelm people having little time for socializing. Therefore, effective mediation strategies should intelligently coordinate all the members in a family community based on specific criteria, such as location, time of day, and the available communication media to support the interaction. This necessarily implies that such mediation process should be adapted to both, the individual's interests and those shared among groups of his/her community. Besides, a mediator system should not be too proactive, since people will eventually refuse to react when there is no urgency, and therefore would not respond in a really important situation. In this interaction scenario, understanding the social and technological context of the involved people is fundamental to ensure the success of the social mediation process.

As a first attempt to tackle the social personalization issues inherent to the proposed communication mediator, we conducted an interview study in cross-generational families. We had a particular interest in understanding communication practices from and toward the elderly, as well as identifying the perceived issues by the latter in a digital communication scenario. By acquiring such knowledge, we identified an initial set of variables that characterize communication asymmetry in cross-generational family communities. We synthesized these findings into a model, aiming to provide social personalization when mediating the communication process between two people. Then, we derived a set of computer-based mediation strategies aiming to connect family members and favoring the eInclusion of the elderly.

Our proposal suggests that the identified asymmetries can be covered by aligning preferences in different levels: communication media, socializing capability, availability for 
socializing, and routine flexibility. Besides, asymmetries in the social link between two people also shape how the mediation process needs to be modeled, especially in terms of who will be the initiator and how long the mediation will take. If the asymmetries between them turn to be too large to be resolved solely by both parties, the introduction of a third family member into the communication process, acting as a communication broker, would be necessary.

The rest of the article is structured as follows. Section 2 reviews recent literature on family connection from a HCI perspective, and discusses the role of technology as impeller of eInclusion in older adults as well as the issues that need to be overcome. Next section presents and formalizes the model proposed to address asymmetries in crossgenerational family communication. Section 4 shows how computer-based mediation strategies can be derived from the proposed model. Finally, section 5 concludes and provides future research directions.

\section{Related Work}

With the proliferation of social media and ubiquitous technology for communicating with family and friends, it is likely that older adults face increasing challenges when interacting with their younger relatives, who typically use those kinds of supporting technology to socialize $[16,17]$. In fact, while most family members desire to enhance their communication with at least one relative, literature suggests that in practice this process is difficult to achieve due to social or technological concerns [28].

When looking deeper into family communication practices, some forms of interaction do not necessarily involve an explicit sharing of messages between older adults and their close family members, but rather an ongoing awareness of the other party's communication state [24]. In other words, people use both personal and environmental cues to help them understand what is happening to the other communication party. Furthermore, Lindley [18] found that elders usually prefer a prolonged contact, which is typically offered by synchronous media (e.g. through face-to-face or phone communication); in turn, asynchronous communication offers advantages to facilitate intergenerational exchanges, such as adapting communication time to a schedule, and providing control over how much effort is dedicated to this kind of interaction.

The eInclusion of older adults through social media or social networking services can effectively assist the integration of the elderly to their families [6]. In addition, it also empowers them with social engagement and self-expression tools [29]. There is also evidence that one of the main benefits of social media usage by older adults is the possibility to enhance their social linking with younger family members, which eventually tends to be appreciated by both parties [23]. Indeed, Bell et al. [3] found that older adults who actively use Facebook state that their main reason to use the platform is to stay connected with their families. 
Unfortunately, most older adults do not feel capable of using digital media [13], and therefore are not able to benefit from them. Therefore, when designing software that support social interaction and social presence for families (particularly if they include older adults), there is an explicit need to consider face-to-face interaction, provide presence awareness mechanisms, assume heterogeneous preferences of social media, allow the mutual social interaction, and properly address usability and accessibility concerns [19].

Several efforts have been done in order to bridge this interaction gap. For instance, Cao et al. [4] identified design implications for facilitating family communication when its members are located in different time zones. Baecker et al. [2], Cornejo et al. [7], Garattini et al. [10], Judge et al. [15], Lindley [18], Muñoz et al. [20], and Rodríguez et al. [25] have adopted a different approach to dial wit that challenge. They designed specific domestic media spaces where remote family members, particularly older adults, can connect with each other using video-mediated communication and others kinds of messaging mechanisms. These social media spaces aim to integrate older adults into their families, and also reduce and prevent eventual negative effects of social isolation.

In summary, the literature indicates that these asymmetries can indeed jeopardize communication among members in a family community. Furthermore, since different generations have different preferences regarding what media they are able or willing to use under a particular social context, it is necessary to follow a personalized approach when facilitating and/or mediating communication between two family members. This is particularly relevant when communication is targeted to be performed with older adults, since further restrictions limit the ways in which the mediation process can be conducted. By actively considering the needs and concerns of older adults into the design of computerbased strategies that mediate asymmetries in family communication, software designers can conceive usable and accessible services that would naturally help enhance the technology appropriation by the elderly, thus favoring their eInclusion.

\section{A Model to Mediate Asymmetries in Family Communication}

In this section we first present the methodology followed to collect the information used to build the model. Then, we present and describe the formal model proposed to mediate asymmetries in family communication. This model intends to support social interaction among members of middle-class family communities living in urban areas, in Chile. The model particularly considers the interactions from/to older adults. Possible extensions to this model and its application to other social realities are part of the future work.

\subsection{Data Collection Methodology}

In order to identify not only asymmetries in cross-generational family communication practices, but also the features that characterize a family community, we used two main 
data sources: (1) a literature review of the most recent systems designed to enable and facilitate family communication, particularly when they involve older adults, and (2) a qualitative interview study.

We conducted semi-structured interviews with the members of 20 cross-generational middle-class families living in urban settlements. For convenience reasons, we focused our study in the metropolitan area of Santiago, Chile and we followed a snowball sampling strategy to recruit the participants. In each family we interviewed three members: an older adult, an adult, and a teenager. Out of the 60 participants, 25 were men $(42 \%)$ and 35 were women (58\%). The interviews were held at the participants' homes. Beforehand, we conducted a small-scale pilot study with three families in order to identify and resolve wording and ambiguous statements in the interview script.

In order to identify emerging themes on cross-generational asymmetries in family communication, we followed the grounded theory approach. Indeed, this resonates with current recommendations and research trends in human-computer interaction [1]. Each interview was tape-recorded with the explicit, free and informed consent of each participant. They were later transcribed, processed through open, axial and selective coding, and analyzed by the authors.

By contrasting the obtained findings with the existing literature, we built a model covering the main characteristics, issues, and social expectations of the stakeholders involved in a family community scenario. Next section presents and formalizes the proposal following the model-driven engineering approach.

\subsection{Family Communication Metamodel}

Communication in family communities can be represented as a metamodel, where each particular family is an instance of such abstract structure. This conception adheres to the model-driven engineering approach [27], and it can be easily implemented using existing tools, such as Eclipse Process Framework [9]. Figure 1 shows the UML class diagram that represents the metamodel.

This representation considers a family as composed at least by a member. Each member has interaction preferences (e.g., preferred tools to conduct synchronous and asynchronous communication), emotional status (set of emotions detected during a certain time window) and eventually behavioral patterns that determine the way in which a person is going to behave under particular circumstances. Every pair of members in a family community has a relationship, which includes several variables that range from the affective attachment among them, to their formal relatedness. There could be an implicit and/or explicit interaction agreement between these pairs, which establishes the interaction frequency and also the time space and digital media involved in these interactions. Based on these agreements, and also in the emotion status of each member, it is possible to determine interaction needs, which represent people that are currently in need of emotional support. 


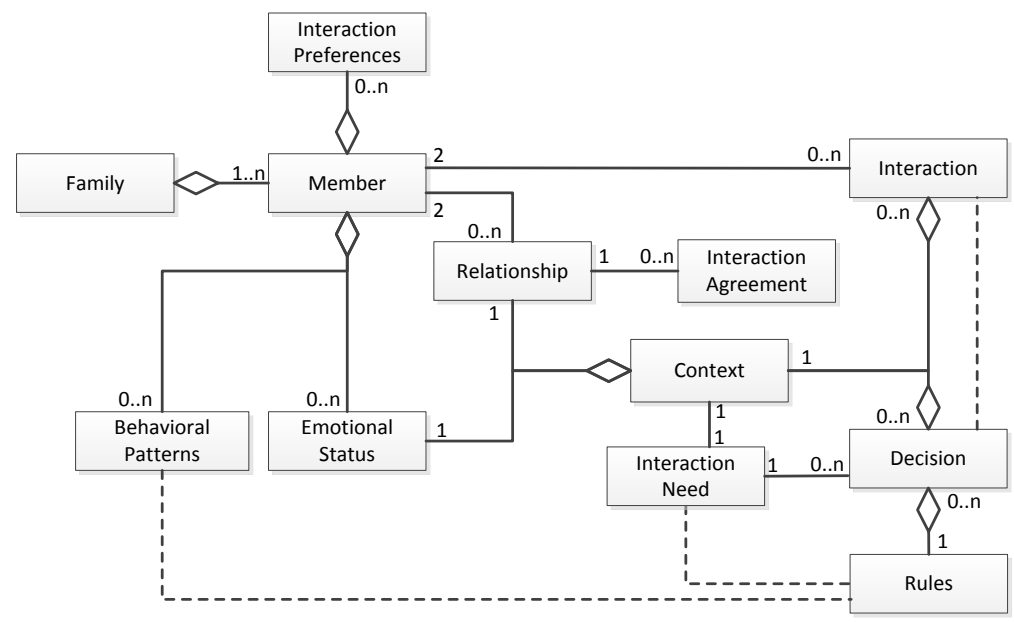

Fig. 1. Family Communication Metamodel

Every interaction need has a context that determines who, how and when other family members could deal with such emotional support. Provided that various family members can potentially intervene in that situation, and trying to not overloading all of them, one or more decisions should be made concerning who will be encouraged to provide support to the member in need. The decision process can be repeated until getting a successful result or using up all the available alternatives.

Decisions are made using a set of rules that indicate how to intervene a relationship considering the social needs of the involved people and their behavioral patterns. The decision process also considers the historical record of interactions between these people. Each decision is translated into particular actions that are made by the system mediating the communication among family members; e.g., an invitation message can be received by a person for contacting other family member that is currently in need of external support. Such an action can be materialized in an interaction, or eventually it can fail. In the latter case, a new decision could be made. Next section describes the process that each particular family communication model uses in order to promote the social interaction from/to older adults.

\subsection{Processing the Family Communication Model}

This process involves four uncoupled stages: data gathering, monitoring, decision- making, and intervention (Fig. 2). Rectangles represent classes of the family model described in the previous section, while rounded rectangles represent processes. Next we explain each model component. 


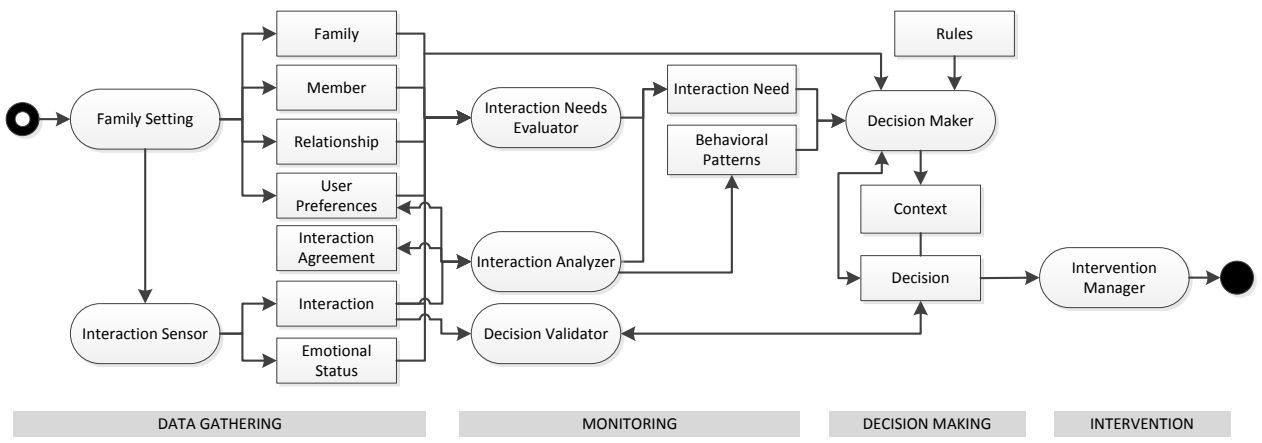

Fig. 2. Basic architecture of the model processing

Data gathering. This stage is in charge of obtaining the basic data of the system; e.g. family composition, user preferences and their interaction agreement. This information is provided through a family setting process, which is performed when the community is created. There is also information that is not provided by the end-users (i.e. family members), but that is automatically captured by the system, and also used as input. Examples of this information are the current emotional status of a family member, or the interactions performed by the participants in a community. Various social media tools, like SocialConnector [21], can act as interaction sensors capturing and recording this information.

Monitoring. This stage adds meaning to the basic information captured in the previous stage. The system tries to determine if there are new interaction needs that should be addressed. Particularly, the interaction needs evaluator analyzes the basic information of family members as well as their emotional status to determine if there is a new need for social support. If it does, the process records such need in the system, and then other components are going to address it. Such need can also be identified by comparing the interaction agreement between each pair of members, and their effective interaction record. The analysis of interactions is also used to determine or adjust behavioral patterns of a user or his/her interaction preferences. The interaction analyzer is the process in charge of performing these activities. Finally, the decision validator process tries to determine if an effective interaction recorded by the system is the result of a stimulus triggered by the system to a user; i.e., a decision made by the system to promote the interaction between two people. If it does, the result is recorded and then used to make future decisions.

Decision-making. This stage takes each interaction need recorded in the communication model, and based on the behavioral patterns and the basic information gathered in the first stage, determines a set of zero or more actions that could be taken to support the people in need. These actions can either be to trigger participation using persuasive strategies, or to raise an alarm and consequently provide awareness on the possible need to family mem- 
bers. The decision maker is the process that determines which decision will be made in each situation. Such process uses a set of rules as support, and makes one or more decisions. Each decision represents a stimulus to a user. The information about these decisions and also about the context in which they were made, is properly recorded in the system.

Intervention. In this stage, the intervention manager processes each decision and acts accordingly. A specific action is made by the system per each decision (e.g. send an email, or show an awareness component to the user). The actions can be successful or not. Regardless the results of an action, such an action and its result are properly recorded in the model, because they are then used to improve the decision-making process.

Fig. 3 shows the software architecture of a social media tool that uses the proposed model to promote interactions among family members, mainly from/to older adults. In this case, we indicate how the model can be embedded into the SocialConnector tool [21] to reach such a goal. SocialConnector, as most social mediators, allows family members to interact using communication mechanisms like videoconferences (through Skype), emails and sharing contents (through Facebook). However, these tools do not identify when a person is in need of external support nor persuade other family members to help reduce or mitigate such situation. That role can be played by the proposed intervention model, which is complementary to the existing tools and can be added as an extension.

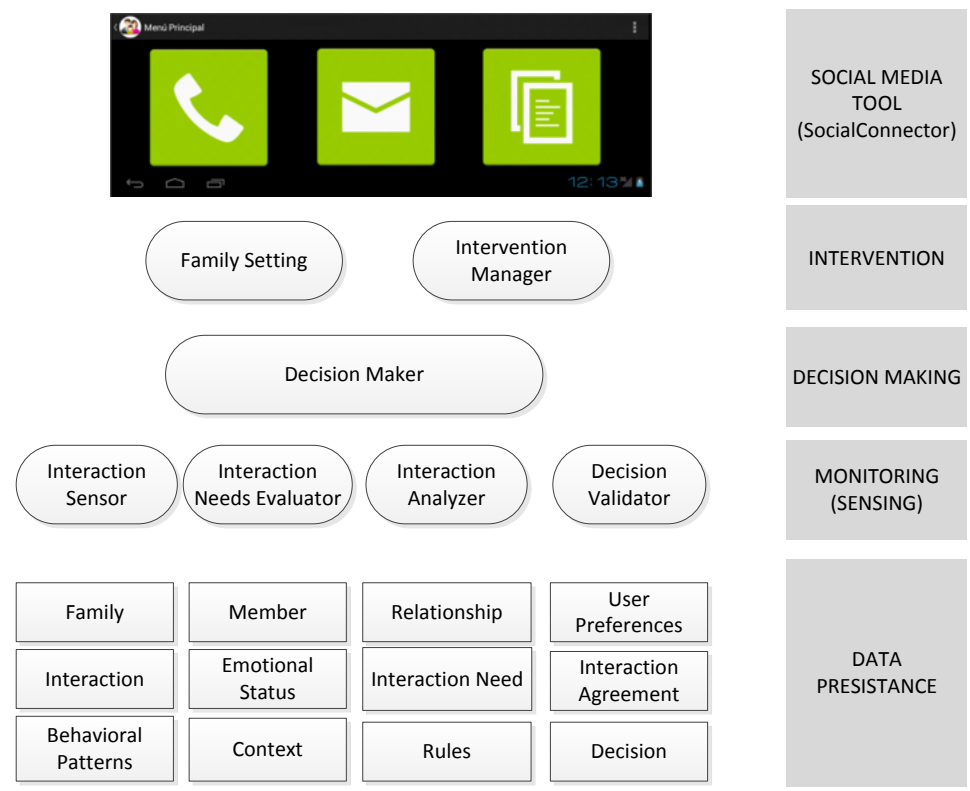

Fig. 3. Architecture of a social media tool that uses the proposed model 
As most modern software tools, we propose to separate the design concerns using layers. In this case, the software extension should involve four layers: data persistence, monitoring, decision-making and intervention. This matches with the stages of the process used to compute the model (Fig. 2). The components and roles of each layer are also those mentioned for the model processing.

\section{$4 \quad$ Using the Model}

In order to illustrate the use of this model as a support for SocialConnector, let us consider a family community composed by twelve members: two older adults (OA), four adults (Ad) and six young people (YP). Considering the relationship existing between them (in terms of social interaction), we can build an interaction graph similar to the one shown in Fig. 4. The different types of links indicate how strong is the affective relationship between each pair of nodes (i.e., between two family members).

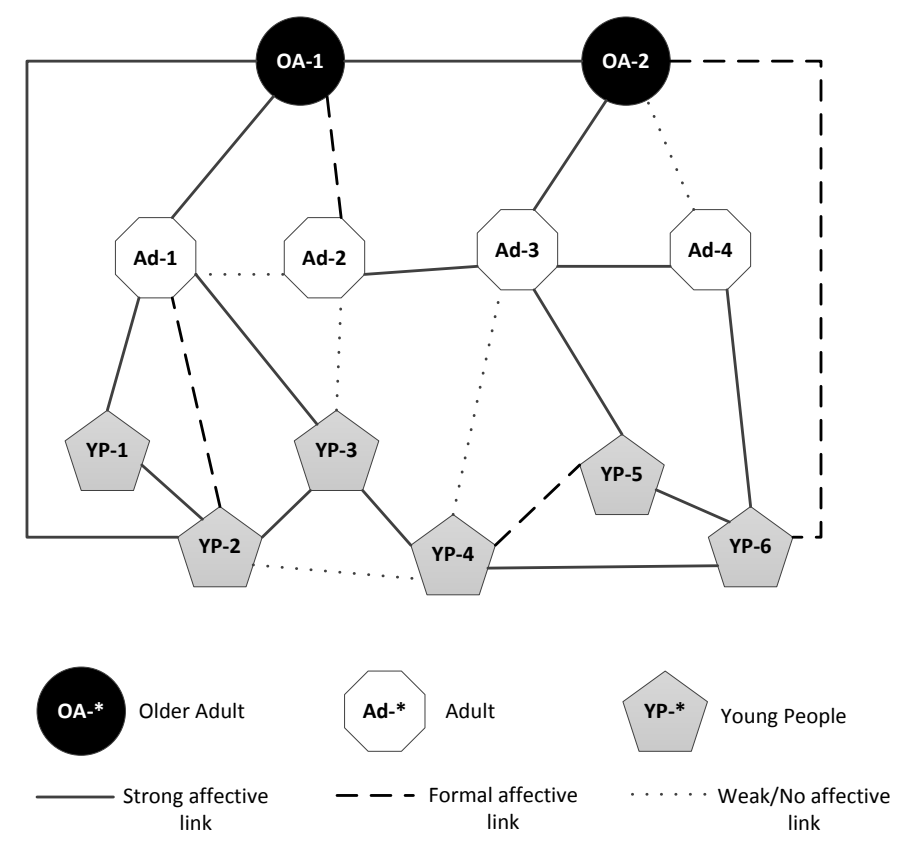

Fig. 4. Example of a simplified family community graph

Let us suppose that the system detects that OA2 has been with a negative mood during the whole morning. Such detection is done by the interaction needs evaluator (Fig. 2 and 3 ), which creates and records a new need in the persistence layer (i.e., it creates a new 
instance of the class interaction need). Then, the decision maker component should determine, based on the set of available rules and the context describing that situation, how to support the person in need. By analyzing the graph, we can see that Ad-3 and OA-1 are the closest family members for OA-2. Probably, the first priority should be to try contacting Ad-3 because adult people usually have better capabilities to deal with problems than older adults. However, Ad-3 would be the first option only if the interaction agreement between Ad-3 and OA-2, and also their interaction preferences, are aligned. In other case, other options must be analyzed.

Once the model determines the most suitable family member to help in this situation, it should make one or more decisions. The decisions are stored in the corresponding class of the model. Each decision triggers an action that the system must perform autonomously in order to persuade the chosen member (e.g., Ad-3) to help the person in need. These actions could be either sending an email or instant message to Ad-3 informing such a situation. The intervention manager component is in charge perform these actions. The result of each action should be verified or guessed (in the worst case) by the system, in order to determine if new decisions should be made because all of the stimuli to Ad-3 failed. Thus, the system uses the model to determine second options, and so on until exhaust all the available intervention alternatives, or eventually succeed.

\section{Conclusion and Future Work}

Asymmetries are inherent to family communication, particularly when they involve intergenerational members. While some people perceive their ability to adapt to other's preferences as natural in terms of communication media and flexibility, external mediation usually needs to be performed in order to increase the chances of effective communication.

After conducting a literature review and a qualitative interview study, we have identified asymmetry sources related to: media preference, socializing capability, the availability of both parties, and their flexibility for performing social interaction. The quality and strength of the affective tie between the involved parties is also relevant when studying family communication. In particular, if the asymmetries between two people appear to be quite strong, the inclusion of a third person acting as a broker in the mediation process is recommended, as it is already naturally considered in family settings.

By providing effective mechanisms to coordinate and mediate social communication among family members through digital means, the elderly appear to show a better reaction to digital media. This facilitates not only their social inclusion to their families, but also to their acceptance and appropriation of ICTs.

As future work we are embedding this model (and its processing) into the SocialConnector system [21]. Then, we will evaluate in the field the model performance through empirical studies that would allow us to determine its impact, as well as gathering feedback to improve it. 
Acknowledgements. This work has been partially supported by the Fondecyt Project (Chile), grant: 1150252. The work of Francisco J. Gutierrez has been supported by the Ph.D. Scholarship Program of Conicyt Chile (CONICYT-PCHA/Doctorado Nacional/2013-21130075).

\section{References}

1. Adams, A., Lunt, P., Cairns, P.: A qualitative approach to HCI research. In: Cairns, P., Cox, A. (Eds.): Research Methods for Human-Computer Interaction. Cambridge, United Kingdom (2008)

2. Baecker, R., Sellen, K., Crosskey, S., Boscart, V., Barbosa Neves, B. Technology to reduce social isolation and loneliness. In: Proc. ASSETS'14, Rochester, USA. ACM Press, 27-34 (2014)

3. Bell, C., Fausset, C., Farmer, S., Nguyen, J., Harley, L., Fain, W.B.: Examining Social Media Use Among Older Adults. In: Proc. HT'13, Paris, France. ACM Press, 158-163 (2013)

4. Cao, X., Sellen, A., Bernheim Brush, A.J., Kirk, D., Edge, D., Ding, X.: Understanding Family Communication across Time Zones. In: Proc. CSCW'10, Savannah, USA. ACM Press, 155158 (2010)

5. Carmichael, A.: Style Guide for the Design of Interactive Television Services for the Elderly Viewers. Independent Television Commission. Winchester, United Kingdom (1999)

6. Cornejo, R., Tentori, M., Favela, J.: Ambient Awareness to Strengthen the Family Social Network of Older Adults. Computer Supported Cooperative Work 22, 309-344 (2013)

7. Cornejo, R., Tentori, M., Favela, J.: Enriching in-person encounters through social media: A study on family connectedness for the elderly. International Journal of Human-Computer Studies 71(9), 889-899 (2013)

8. Czaja, S.J., Charness, N., Fisk, A.D., Hertzog, C., Nair, S.N., Rogers, W.A., Sharit, J.: Factors Predicting the Use of Technology. Psychology \& Aging 21, 333-352 (2006)

9. The Eclipse Foundation. Eclipse Process Framework Project (EPF). Available at: http://eclipse.org/epf/. Last visit: Dec. 29, 2014

10. Garattini, C., Wherton, J., Prendergast, D.: Linking the lonely: an exploration of a communication technology designed to support social interaction among older adults. Universal Access in the Information Society 11(2), 211-222 (2012)

11. Giddens, A.: Conversations with Anthony Giddens: Making Sense of Modernity. Stanford University Press, Palo Alto (1998)

12. Hawthorn, D.: Possible Implications of Aging for Interface Designers. Interacting with Computers $12,151-156(2000)$

13. Hope, A., Schwaba, T., Piper, A.M.: Understanding Digital and Material Social Communications for Older Adults. In: Proc. CHI'14, Toronto, Canada. ACM Press, 3903-3912 (2014)

14. House, J.S.: Social Isolation Kills, but How and Why? Psychosomatic Medicine 63, 273-274 (2001)

15. Judge, T.K., Neustaedter, C., Harrison, S.: Inter-Family Messaging with Domestic Media Spaces. In: Neustaedter, C., Harrison, S., Sellen, A. (Eds.): Connecting Families, pp. 141-157. Springer-Verlag, London (2013) 
16. Lindley, S.E., Harper, R., Sellen, A.: Designing for Elders: Exploring the Complexity of Relationships in Later Life. In: Proc. British-HCI'2008, Liverpool, United Kingdom. British Computer Society, 77-86 (2008)

17. Lindley, S.E., Harper, R., Sellen, A.: Desiring to Be in Touch in a Changing Communications Landscape: Attitudes of Older Adults. In: Proc. CHI'09, Boston, USA. ACM Press, 1693$1702(2009)$

18. Lindley, S.E.: Shades of Lightweight: Supporting Cross-generational Communication Through Home Messaging. Universal Access in the Information Society 11(1), 31-43 (2012)

19. Moser, C., Fuchsberger, V., Neureiter, K., Shellner, W., Tscheligi, M.: Elderly's Social Presence Supported by ICTs: Investigating User Requirements for Social Presence. In: Proc. SocialCom'11, Boston, USA. IEEE Press, 738-741 (2011)

20. Muñoz, D., Cornejo, R., Gutierrez, F.J., Favela, J., Ochoa, S.F., Tentori, M.: A Social CloudBased Tool to Deal with Time and Media Mismatch of Intergenerational Family Communication. Future Generation Computer Systems. In press. Available at: http://dx.doi.org/10.1016/j.future.2014.07.003 (2014)

21. Muñoz, D., Gutierrez, F.J., Ochoa, S.F., Baloian, N.: SocialConnector: A Ubiquitous System to Ease the Social Interaction Among Family Community Members. Accepted in the International Journal of Computer Systems, Science \& Engineering. 2014.

22. Neal, A.G., Collas, S.F.: Intimacy and Alienation: Forms of Estrangement in Female/Male Relationships. Garland Publishing, New York (2000)

23. Nef, T., Ganea, R.L., Müri, R.M., Mosinmann, U.P.: Social Networking Sites and Older Users - A Systematic Review. International Psychogeriatrics 25(7), 1041-1053 (2013)

24. Riche, Y., Mackay, W.: PeerCare: Supporting Awareness of Rhythms and Routines for Better Aging in Place. Computer Supported Cooperative Work 19, 73-104 (2010)

25. Rodríguez, M.D., Gonzalez, V.M., Favela, J. Santana, P.C.: Home-based communication system for older adults and their remote family. Computers in Human Behavior 25(3), 609-618 (2009)

26. Roupa, Z., Nikas, M., Gerasimou, E., Zafeiri, V., Giasyrani, L., Kazitori, E., Sotiropoulou, P.: The Use of Technology by the Elderly. Health Science Journal 4(2), 118-126 (2010)

27. Schmidt, D.C.: Model-Driven Engineering. IEEE Computer 39(2) 25-31 (2006)

28. Tee, K., Brush, A.J.B., Inkpen, K.M.: Exploring Communication and Sharing Between Extended Families. International Journal of Human-Computer Studies 67, 128-138 (2008)

29. Waycott, J., Vetere, F., Pedell, S., Kulik, L., Ozanne, E., Gruner, A., Downs, J.: Older Adults ad Digital Content Producers. In: Proc. CHI'13, Paris, France. ACM Press, 39-48 (2013) 